University of Nebraska - Lincoln

DigitalCommons@University of Nebraska - Lincoln

\title{
A Novel Cost-Efficient On-Line Intermediate Waveband-Switching Scheme in WDM Mesh Networks
}

\author{
Mengke Li \\ University of Nebraska-Lincoln \\ Wang Yao \\ University of Nebraska-Lincoln \\ Byrav Ramamurthy \\ University of Nebraska-Lincoln, bramamurthy2@unl.edu
}

Follow this and additional works at: https://digitalcommons.unl.edu/cseconfwork

Part of the Computer Sciences Commons

Li, Mengke; Yao, Wang; and Ramamurthy, Byrav, "A Novel Cost-Efficient On-Line Intermediate WavebandSwitching Scheme in WDM Mesh Networks" (2005). CSE Conference and Workshop Papers. 75.

https://digitalcommons.unl.edu/cseconfwork/75

This Article is brought to you for free and open access by the Computer Science and Engineering, Department of at DigitalCommons@University of Nebraska - Lincoln. It has been accepted for inclusion in CSE Conference and Workshop Papers by an authorized administrator of DigitalCommons@University of Nebraska - Lincoln. 


\title{
A Novel Cost-Efficient On-Line Intermediate Waveband-Switching Scheme in WDM Mesh Networks
}

\author{
Mengke Li, Wang Yao, and Byrav Ramamurthy \\ Department of Computer Science and Engineering, \\ University of Nebraska-Lincoln, Lincoln, NE 68588-0115, USA. \\ Email: \{mli,wyao,byrav\}@cse.unl.edu
}

\begin{abstract}
Waveband switching (WBS) is an important technique to save switching and transmission cost in wavelength -division multiplexed (WDM) optical networks. A cost-efficient WBS scheme would enable network carriers to increase the network throughput (revenue) while achieving significant cost savings. We identify the critical factors that determine the WBS network throughput and switching cost and propose a novel intermediate waveband switching (IT-WBS) algorithm, called the minimizing-weighted-cost (MWC) algorithm. The MWC algorithm defines a cost for each candidate route of a call. By selecting the route with the smallest weighted cost, MWC balances between minimizing the call blocking probability and minimizing the network switching cost. Our simulations show that MWC outperforms other wavelength/waveband switching algorithms and can enhance the network throughput at a reduced cost.

Index Terms - Optical fiber network, wavelength division multiplexing, waveband, switching, routing, blocking probability.
\end{abstract}

\section{INTRODUCTION}

Optical wavelength-division multiplexed (WDM) networks are regarded as the most promising candidates for the next-generation backbone networks. Waveband switching (WBS) is introduced as a cost saving technique for wide-area WDM networks [1]-[7]. It groups a specific set of wavelengths into a waveband at a grouping node. The grouped wavelengths are transmitted as a single bundle along some common links. The waveband is disaggregated back into wavelengths at a disaggregating node. A waveband-route is formed along these common links between the grouping and the disaggregating nodes. No optic- electronic conversion is required along such a waveband-route, which incorporates all-optical transmission. On the other hand, the traditional wavelength-routed WDM networks route each wavelength separately in a lightpath, which occupies an input and an output switching ports at each node along the route. Thus, adopting WBS reduces the number of ports utilized [1][2]. Since the switching and transmission costs in the optical domain of a WDM network are dominated by the number of occupied OXC ports, the WBS technique has the potential of saving significant costs.

In a real-world network, dynamically arrived traffic may be blocked due to lack of resources. The call blocking probability reflects the network throughput, which affects the revenue. In addition, provisioning a call in a WBS network is constrained by both the waveband availability and the wavelength availability in a waveband. These constraints, which are influenced by the efficiency of the waveband grouping strategy, also affect the network performance in terms of call blocking probability. Thus, it is important to design a cost-efficient waveband-grouping scheme that can improve or maintain the network throughput with reduced operational costs.
Previous studies verified the benefits of adopting the WBS technique [1]-[3]. Some other studies aimed at achieving maximum cost savings with WBS [4]-[7]. Most of the studies group calls with the same source-destination pair into a waveband, which is referred to as end-to-end waveband switching (ETE-WBS). The ETE grouping constraint can be eliminated. Instead, intermediate waveband switching (IT-WBS), which groups calls at an intermediate node and switches them in a waveband-route along some common links, can further reduce the operational costs. The advantage of IT-WBS over ETE-WBS in cost savings is illustrated and analyzed in [7]. However, the simple IT-WBS scheme in [7] did not show good performance in terms of call blocking probability. It is challenging to design a WBS scheme that can increase the network throughput at a reduced cost.

We address the problem of designing an IT-WBS algorithm in WBS networks under dynamic traffic. The objective is to achieve a low call blocking probability at a reduced operational cost. To the best of our knowledge, no previous work has addressed this issue. We propose an on-line IT-WBS algorithm, called minimizing-weighted-cost (MWC) algorithm. To achieve the goal, MWC incorporates three key factors into the routing decisions, viz. the operational cost, the path length, and the utilization of active waveband-routes. By combing these three factors, a weight is calculated for a route. The decision of provisioning calls along the route is made based on the weight of the route. Additionally, when and where to set up a new waveband-route influence the performance of a WBS network. MWC takes into considerations of the possible utilization of the waveband-route and its path length when making the decision.

\section{Network Model AND PROBlem StATEMENT}

A homogeneous WBS network is considered, where each node is capable of WBS with fixed fiber capacities in terms of wavelengths and wavebands. The problem is to design a WBS algorithm with routing, wavelength/waveband assignment, and waveband grouping under dynamic traffic requests.

\section{A. Network node architecture}

Besides its cost-efficient, adopting WBS can reduce the complexity of optical crossconnects (OXCs). While defining a label switched waveband-path in a generalized MPLS framework was presented in [8] and is in progress, the architecture of an OXC in traditional WDM networks should also be modified to support WBS. A typical architecture of a WBS-enabled OXC is shown in [1][6] termed a multi-granular optical crossconnect (MG-OXC). This work assumes that each node in the network is an MG-OXCs, which is the same as the 
one in [2]. A waveband-path along the MG-OXCs can reduce the total number of ports used. The efficiency of the WBS method in terms of port saving ratio is illustrated in [4][5]. If all ports in the MG-OXCs have the same cost, the number of used ports can represent the routing cost in the optical domain. The real cost savings can also be derived from the saving of occupied MG-OXC ports.

\section{B. Waveband grouping strategies}

The waveband grouping strategy could be classified as an end-to-end grouping [1] and an intermediate grouping [3][7]. WBS algorithms with different grouping strategies can achieve different performance. The advantage of IT-WBS over ETE-WBS in cost savings can be illustrated using an example shown in Fig.1. There are three calls, $\lambda_{1}, \lambda_{2}$, and $\lambda_{3}$, originating from different source nodes and destined to the same destination node. Through IT-WBS, the three calls can be grouped into a waveband-route $B_{I}$ at an intermediate node $S$. In this way, it reduces the number of ports used at wavelength crossconnect (WXC) stage from node $S$ to node $D$. On the contrary, the three calls cannot be grouped into a waveband-route through ETE-WBS. Thus, IT-WBS reduces more number of ports used than ETE-WBS does in this situation.

In both types of WBS, waveband granularity, which is the maximum number of wavelengths in a waveband, is an important factor in achieving optimal performance. Different types of traffic require different waveband granularities to achieve maximum cost savings. Under dynamic traffic request, the current optimal configuration of waveband granularities may not be optimal in future. Thus, it is more suitable to dynamically group wavelengths into a waveband.

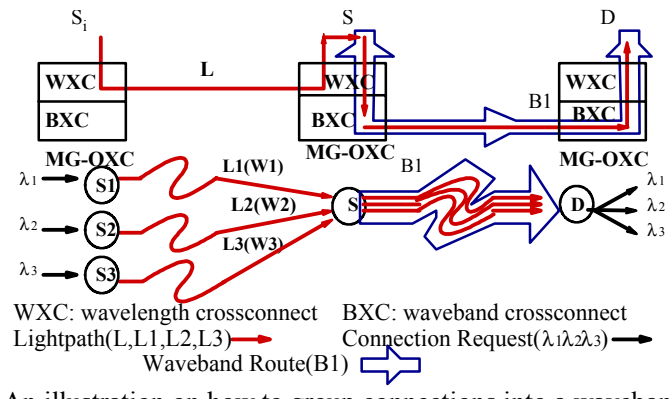

Fig.1. An illustration on how to group connections into a waveband at an intermediate node.

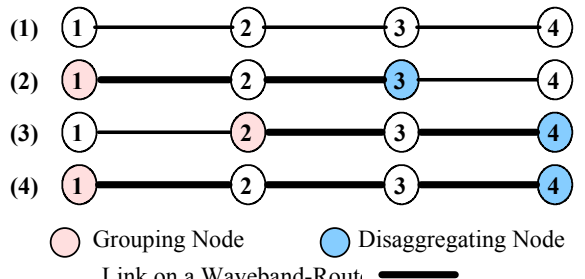

Fig. 2. Collecting available waveband-routes along a 3-hop path.

\section{Problem statement}

Given the network configuration, the Dynamic Waveband Switching (DWBS) problem is to provision a call upon its arrival such that the overall network throughput can be improved at a reduced operational cost. The network configuration includes the followings.

1. The network topology $G(V, E)$.

2. The fiber capacity $(W)$.

3. The waveband granularity $(G)$.

4. The maximum number of active (used) wavebands in a fiber $(B=\lceil W / G\rceil)$

5. The current wavelength availability, the current waveband availability.

6. The utilization of the current active waveband-routes.

We assume that each call requires a whole wavelength capacity and there is no fiber switching. Moreover, the network configuration is specified by the utilized MG-OXCs, each of which has $B$ input/output waveband-switching ports, $B$ waveband multiplexers/demultiplexers, and $W$ input/output wavelength-switching ports. The network configuration can be expressed as $G_{0}\left(V_{0}, E_{0}, W, B, G\right)$, where $V_{0}$ is its node set, $E_{0}$ is its link set, $W$ is the set of wavelengths on each fiber, $B$ is the set of wavebands on each fiber, and $\mathrm{G}$ is the waveband granularity.

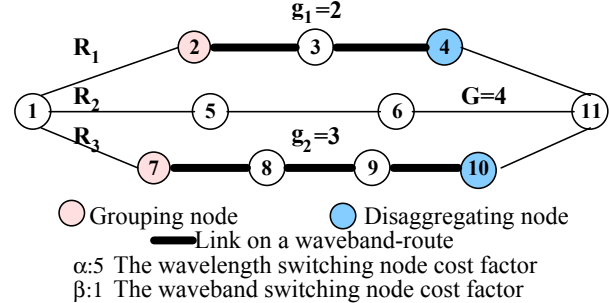

Fig. 3. An example of a call $\lambda$ with three candidate routes, $R_{1}, R_{2}$, and $R_{3}$. Route $\mathrm{R}_{1}$ traverses along a two-hop waveband-route. Route $\mathrm{R}_{3}$ traverses along a three-hop waveband-route.

\section{MinIMIZING-WeIGHTED-Cost INTERMEDIATE WAVEBAND-SWITCHING SCHEME}

In this section, we propose the minimizing-weight-cost (MWC) scheme to address the DWBS problem. To achieve a low call blocking probability at a reduced operational cost, MWC considers three factors when selecting a route for a call, viz. the operational cost, the path length, and the utilization of active waveband-routes.

The operational cost combined with the call blocking probability, which is determined by the length of the route and the utilization of the traversed waveband-route in a WBS network, is important for the network carriers to achieve optimal revenue. The MWC scheme balances the three factors when routing a call, based on which a weighted cost is assigned to a route. First, to minimize the cost, a call prefers a waveband-route to a lightpath. However, adopting a waveband-route at an intermediate node may increase the path length substantially. Moreover, defining the operational cost of a call is very complex in a real-world network, where we abstract it as the occupied $\mathrm{O} / \mathrm{O} / \mathrm{O}$ ports and occupied $\mathrm{O} / \mathrm{E} / \mathrm{O}$ ports along the route. Second, to avoid wasting the bandwidth of a waveband-route, a call prefers an active waveband-route with the least free spaces. In addition, the higher utilization of a waveband-route is, the more savings can be obtained. This is straightforward and is shown in [1]. Third, a call prefers a shorter route to a longer route because a shorter route usually has lower blocking probability than a longer one. Furthermore, a shorter waveband-route may have higher utilization than a 
longer one, since more calls may traverse along it. However, the longer the waveband-route is, the more cost savings can be achieved. In summary, these three factors are influenced by each other and thus a balance between them is important.

For each call, a set of candidate routes is collected, among which the one with the minimum weighted cost is selected to achieve optimum overall performance. The candidate routes are lightpaths, which may traverse through some intermediate waveband-routes. To obtain a set of candidate routes for a call, the MWC scheme first searches for up to k-shortest paths with available wavelengths. It then searches for all possible intermediate waveband-routes along these paths. Finally, MWC calculates the weight for each of the routes. Fig.2 illustrates on how to collect all possible waveband-routes along a 3-hop path. A single path decomposition method is introduced here. In addition, according to [1] and [2], a waveband-route with less than 2 hops cannot gain any advantage in cost savings. Thus, a possible waveband-route should have at least 2 hops. Let $\mathrm{k}$ be the number of possible waveband-routes along an $h$-hop path, there is

$$
k=\left(\begin{array}{l}
2 \\
h
\end{array}\right)+\ldots+\left(\begin{array}{l}
h \\
h
\end{array}\right) \text {. }
$$

\section{A. Weighted cost of a candidate route}

When a call dynamically arrives at the network, we defined the weighted cost for a candidate route $p_{i}$ based on the above three factors as

$$
C\left(p_{i}\right)=\frac{\alpha \times N_{E}+\beta \times N_{O}}{H} \times \rho_{s}
$$

where $\alpha$ is the cost factor of an $\mathrm{O} / \mathrm{E} / \mathrm{O}$ port, $\beta$ is the cost factor of an $\mathrm{O} / \mathrm{O} / \mathrm{O}$ port, $N_{E}$ is the total number of occupied $\mathrm{O} / \mathrm{E} / \mathrm{O}$ ports along the route, $N_{O}$ is the total number of occupied $\mathrm{O} / \mathrm{O} / \mathrm{O}$ ports along the route, $H$ is the hop count of the route, and $\rho_{s}$ is the ratio of the number of free wavelengths in the waveband to the waveband granularity. If the route does not traverse along an intermediate waveband-route, $\rho_{s}$ is 1 . Note that a waveband is switched in the optical domain and the waveband-switching ports are OOO ports. On the other hand, a wavelength is switched through OEO ports. As indicated in [4], an OEO port usually costs four times higher than an OOO port. Thus, the value of $\alpha$ is set to 5 and the value of $\beta$ is set to 1 .

After calculating the weighted cost for each candidate route, the MWC scheme selects the one with the smallest weighted cost. We explain how the MWC scheme selects a route through an example shown in Fig. 3. As shown in Fig. 3, there is a call $c<1,11>$ from node 1 to node 11 . The $\mathrm{k}$-shortest path algorithm returns with three paths, which are $p_{I}(1 \rightarrow 2 \rightarrow 3 \rightarrow 4 \rightarrow 11)$, $p_{2}(1 \rightarrow 5 \rightarrow 6 \rightarrow 11)$, and $p_{3}(1 \rightarrow 7 \rightarrow 8 \rightarrow 9 \rightarrow 10 \rightarrow 11)$. Along path $p_{I}$, there is an active waveband route $B_{I}(2 \rightarrow 3 \rightarrow 4)$ from node 2 to node 4 which has already grouped 2 calls. Along path $p_{3}$, there is an active waveband route $B_{3}(7 \rightarrow 8 \rightarrow 9 \rightarrow 10)$ from node 7 to node 10 which has grouped 3 calls.

In this example, let that there is no other candidate route except for the above three. According to (2), the weighted cost of path $p_{l}$ along the waveband-route $B_{l}$ is $\frac{5 \times 2+1 \times 4+5 \times 2}{4} \times \frac{2}{4}=3$. The weighted cost of path $p_{2}$ is 10 . The weighted cost of path $p_{3}$ along the waveband-route $B_{3}$ is 1.3 . Among these candidate routes, $p_{3}$ has the smallest weight. Thus, a lightpath traversing path $p_{3}$ and grouped into the waveband-route $B_{3}$ at node 7 is set up for the call $c<1,11>$, if there is a common free wavelength along path $p_{3}$ and waveband-route $B_{3}$. Otherwise, a lightpath traversing path $p_{1}$ and grouped into the waveband-route $B_{l}$ at node 2 is set up, if there is a common free wavelength along path $p_{1}$ and waveband-route $B_{l}$. Otherwise, a lightpath is set up along path $p_{2}$ for the call $c<1,11>$.

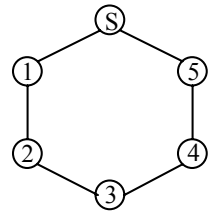

(a) A ring network

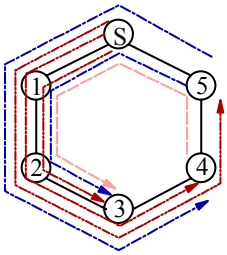

(b) Connections that could route along path $\mathrm{P}_{13}(\mathrm{~s}->1->2)$

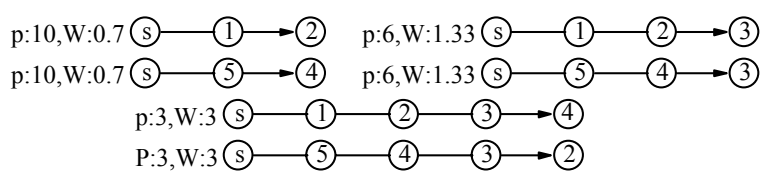

(c) Weights of the paths from $s$ to the rest of the network, where
\[ \alpha=5, \beta=1 \text {, and } \mathrm{G}=4 \text {. } \]

Fig. 4. An illustration on how to calculate the weight for a route when MWC is trying to set up a new waveband-route.

\section{B. Strategies on setting up a new active waveband-route}

Initially, there is no traffic in the network and thus no active waveband-route. When the first call arrives, we need to decide whether and which new wavelength-route or waveband-route should be set up.

There are two factors should be considered when setting up a new waveband-route. One factor is the possible utilization of the waveband-route. The more calls choose the waveband-route, the higher utilization it has, which result more cost savings and the higher throughput along the waveband-route. The other factor is the hop count of the waveband-route. The longer the waveband-route is, the more cost savings can be achieved. However, longer route means a larger blocking probability and smaller throughput. In addition, the longer the waveband-route is, the less calls can choose it. Considering the two factors, we define the weight for a new candidate route $B_{p}$ as

$$
W\left(B_{p}\right)=\frac{\alpha+\beta \times H}{p\left(B_{p}\right)},
$$

where $\alpha$ is the cost factor of an OEO port, $\beta$ is the cost factor of an OOO port, $H$ is the hop count of the route, and $p\left(B_{p}\right)$ is the number of calls in the network that may traverse along the route $B_{p}$. The MWC scheme selects the one with the minimum weight to set up a new waveband-route given enough free resources along the route. Otherwise, no new waveband-route is set up.

An example is presented in Fig. 4 on how MWC determines the setting up of a new active waveband-route. Fig. 4(a) shows the topology of a six-node bi-directional ring network. When a 
call $c_{3}<\mathrm{s}, 3>$ from node $s$ to node 3 arrives at the network, MWC determines whether and which waveband-route should be set up. There are two paths for $c_{3}$, which are the path $p_{1}(s \rightarrow 1 \rightarrow 2 \rightarrow$ 3 ) and path $p_{2}(s \rightarrow 5 \rightarrow 4 \rightarrow 3)$. For path $p_{1}$, there are three candidate waveband-routes, which are $p_{11}(s \rightarrow 1 \rightarrow 2), p_{12}(1 \rightarrow 2$ $\rightarrow 3)$, and $p_{13}(s \rightarrow 1 \rightarrow 2 \rightarrow 3)$. For path $p_{2}$, there are also three candidate waveband-routes, $p_{21}(s \rightarrow 5 \rightarrow 4), p_{22}(5 \rightarrow 4 \rightarrow 3)$, and $p_{23}(s \rightarrow 5 \rightarrow 4 \rightarrow 3)$. MWC calculates the weight for each of the paths using (3). Fig. 4(b) illustrates on how to obtain the number of calls that may traverse along the candidate path $p_{13}(s \rightarrow 1 \rightarrow 2 \rightarrow 3)$. As shown in the figure, the following calls may traverse along $p_{13}$, which are $c(s \rightarrow 1 \rightarrow 2 \rightarrow 3)$, $c(s \rightarrow 1 \rightarrow 2 \rightarrow 3 \rightarrow 4), c(s \rightarrow 1 \rightarrow 2 \rightarrow 3 \rightarrow 4 \rightarrow 5), c(5 \rightarrow s \rightarrow 1 \rightarrow 2 \rightarrow 3)$, $c(5 \rightarrow s \rightarrow 1 \rightarrow 2 \rightarrow 3 \rightarrow 4)$, and $c(4 \rightarrow 5 \rightarrow s \rightarrow 1 \rightarrow 2 \rightarrow 3)$. Thus there are total up to six calls that may route along it. As the hop count of the path $p_{13}$ is 3 , its weight is 1.33 . Fig. 4(c) shows all possible candidate waveband-routes from node $s$ to the rest of the network with accordingly weights. For the call $c_{3}$, there are four routes have the smallest weight of $0.7, p_{11}(\mathrm{~s} \rightarrow 1 \rightarrow 2)$, $p_{12}(1 \rightarrow 2 \rightarrow 3), p_{21}(s \rightarrow 5 \rightarrow 4)$, and $p_{22}(5 \rightarrow 4 \rightarrow 3)$. MWC picks the first one to set up a new waveband-route if there are matches.

\section{MWC algorithm}

The MWC algorithm first adopts Yen's k-shortest path algorithm [9] to obtain a set of candidate paths for a call. It then calculates the weighted cost for each candidate-paths. If there are enough resources, a new waveband-route or a lightpath that may traverse along an intermediate waveband-route, is set up. The algorithm utilizes the first-fit wavelength assignment algorithm (FFWA) [10[11], which assigns the first free wavelengths to the route. If a new waveband-route should be set up, the algorithm utilizes the last-fit waveband assignment algorithm (LFBA), which assigns the least available waveband to the route. Other wavelength assignments and waveband assignments are simulated as well. However, their performance is not better. As wavelength converters are not adopted, each lightpath/waveband-route is constrained by the wavelength/ waveband continuity separately. The detailed description of the algorithm is shown in Fig. 5.

\section{NUMERICAL RESULTS}

We conduct simulations on the NSF network with 14 nodes and 21 bi-directional links, which is the same as shown in [2]. Each node is an MG-OXC. The cost factor is 1 for an OOO port and 5 for an OEO port. Each simulation result is obtained by running 1,000,000 calls. Poisson traffic is generated for the network with a rate $\lambda$. The traffic is uniformly distributed among the connection pairs. The call holding time is exponential with a mean $\mu$. Thus, the network load in terms of Erlang is $L=\lambda / \mu$. The following notations are used in this section. $W$ is the fiber capacity. $G$ is the waveband granularity. $B$ is the maximum number of wavebands in a fiber. $L$ is the network load in Erlang. WRA is the traditional wavelength routing approach with the FFWA assignment and the same routing. The simulations are conducted under different configurations, where $W=\{16,20,24,28,32\}, G=\{2,4,8\}$, and
$L=\{80,85,90,95,100,105\}$. The number of wavebands on a fiber can be obtained from $W$ and $G$.

Fig. 6 compares the blocking probability of the MWC algorithm and the WRA algorithm. As can be seen, MWC can achieve smaller blocking probabilities under various scenarios, which are about 1/3 of WRA. Additionally, the larger the fiber capacity is, the larger the performance gap is. Fig. 8 presents the blocking probability of MWC under different waveband granularities. According to Fig. 7, the blocking probability of MWC slightly increases as the waveband granularity increases. This is reasonable as the probability that many calls can be grouped together is small. If the waveband granularity continues to increase, more wavelengths in the waveband will be left unused and the waveband utilization becomes low.

\section{The MWC Algorithm}

Input: the network configuration and a call.

Output: the updated network configuration

Step 1. search for up to $k$ shortest paths (denoted by $\Phi_{p}=\left\{p_{l}\right.$, $\left.\left.p_{2}, \ldots, p_{k}\right\}\right)$ for the call.

Step 2. set miniCost $\leftarrow \infty, n o B R \leftarrow$ True, $\min B R \leftarrow \Phi$, and $\min W \leftarrow \Phi$.

Step 3. for each path $p_{i} \in \Phi_{p}$

a. search for the candidate waveband-routes (denoted as $\left.\Phi_{B}\left\{B_{1}, B_{2}, \ldots, B_{k}\right\}\right)$.

b. if $k \neq 0$, set $n o B R \leftarrow F A L S E$.

c. for each $B_{j} \in \Phi_{B}$ with waveband $b_{i}$.

i. $\quad$ set $\chi_{s} \leftarrow\left(b_{i}-1\right) * G, \chi_{e} \leftarrow b_{i}{ }^{*} G-1$.

ii. starting from $\chi_{s}$ limited by $\chi_{e}$, search for the first free wavelength along $B_{j}$. Let it be $w_{x}$.

iii. if there is no such free wavelength, return to Step c.

iv. check the availability of $w_{x}$ on the remaining links along $p_{i}$.

v. if $w_{x}$ is not available, set $\chi_{s} \leftarrow w_{x}+1$ and return to Step ii.

vi. calculate cost factor $C\left(p_{i}\right)$ according to (2). To save time, we calculate and store each $\mathrm{p}\left(B_{j}\right)$ beforehand.

vii. if miniCost $>C\left(p_{i}\right)$ set $\min C o s t \leftarrow C\left(p_{i}\right), \min B R \leftarrow B_{j}$ $\min W \leftarrow w_{x}$, and return to Step c.

Step 4. if $n o B R=$ True

$$
\text { set } \min B R \leftarrow \infty \text { and } \min N B R \leftarrow \Phi
$$

for each path $p_{i} \in \Phi_{p}$ with enough free resources calculate $W\left(p_{i}\right)$ according to (3)

if $\min B R>W\left(B p_{i}\right)$, set $\min B R \leftarrow W\left(p_{i}\right)$ and $\min N B R \leftarrow p_{i}$ set up a new waveband-route along $p_{i}$, provision the call along it, update the network accordingly and return.

Step 5. if $\min B R=\Phi$

set up a lightpath along the shortest path.

else set up the lightpath along $\min B R$ with $\min W$. Update the network accordingly.

If both the above attempts fail, the call is blocked.

Fig. 5. Minimizing-weighted-cost intermediate waveband switching algorithm. 


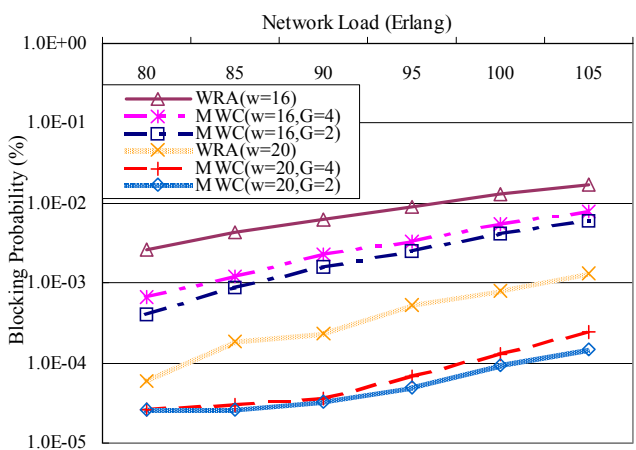

Fig. 6. Performance comparison of the MWC algorithm and the WRA approach on the NSFNet under different network loads in terms of call blocking probability.

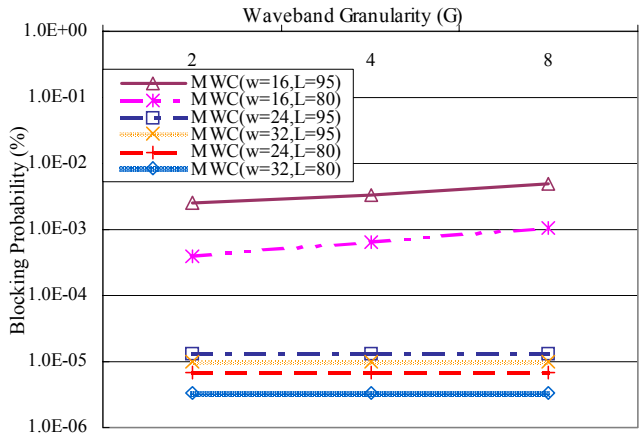

Fig.7. Call blocking probabilities of MWC on the NSFNet under different waveband granularities.

Fig. 8 and Fig. 9 show the cost savings of MWC, which is defined as the ratio of the operational cost difference between the two approaches to the operational costs of WRA. Fig. 8 presents the cost savings of MWC under different traffic loads. As can be seen, the cost savings slightly decreases as the traffic load increases because of the increased blocking of traffic under heavy load. Fig.9 illustrates that the cost savings increase with the fiber capacity. This is because a larger fiber capacity leads to a smaller call blocking probability. In conclusion, MWC can achieve significant cost savings ranging from $25 \%$ to $60 \%$ compared to WRA.

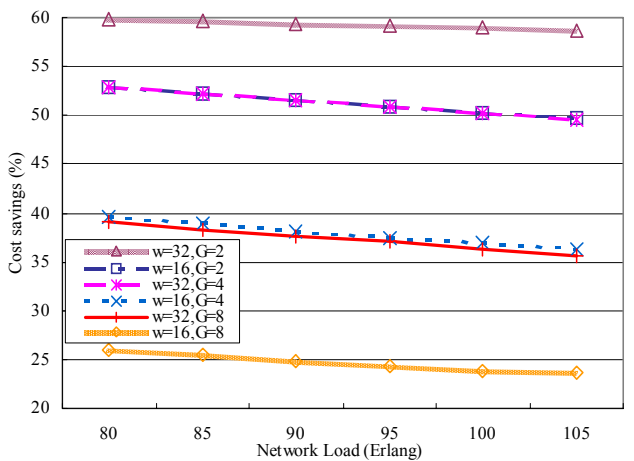

Fig. 8. Cost savings of the MWC algorithm on the NSFNet under different network loads compared with the WRA approach, where $\alpha=5$ and $\beta=1$.

\section{CONCLUSIONS AND FUTURE WORKS}

Intermediate waveband switching (IT-WBS) is a cost- efficient approach to operate WDM networks with WBS function. We proposed the minimizing-weighted-cost IT-WBS algorithm (MWC) to provision dynamic traffic in WDM mesh networks. To achieve minimal call blocking probability with reduced operational costs, MWC considers three key factors into the routing decision for a call. A balancing mechanism for adopting the three factors is utilized, based on which a weighted cost is assigned to a route. The routing decision heavily influences the performance of an IT-WBS network. In addition, MWC determines the setting up of a new waveband-route according to its possible utilization and its path length. Our simulation results show that MWC has lower call blocking probability than the traditional wavelength routing approach with $20 \%$ to $60 \%$ operational cost savings.

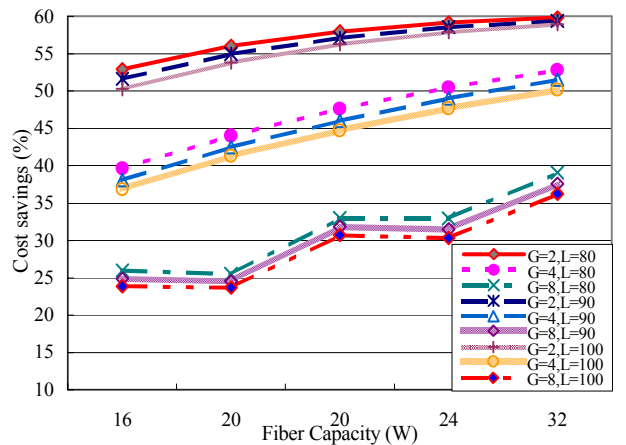

Fig. 9. Cost savings of MWC on the NSFNet under different fiber capacities compared with the WRA approach, where $\alpha=5$ and $\beta=1$.

\section{REFERENCES}

[1] Y. Suemura, I. Nishioka, Y. Maeno, S. Araki, R. Izmailov, and S. Ganguly, "Hierarchical routing in layered ring and mesh optical networks," in Proc. of IEEE ICC, Apr.-May 2002, pp. 2727-2733.

[2] M. Li and B. Ramamurthy, "A generic graph model for dynamic waveband-switching in WDM mesh networks", SPIE/Kluwer Photonic Networks Communication Magazine, Vol. 10, No. 3, pp. 309-331, Nov. 2005.

[3] L. Noirie, M. Vigoureux, and E. Dotaro, "Impact of intermediate grouping on the dimensioning of multi-granularity optical networks," in Proc. of Optical Fiber Commun. Conf., Mar. 2001, pp. TuG3/1-3.

[4] P. Bullock, C. Ward, and Q. Wang, "Optimizing wavelength grouping granularity for optical add-drop network architecture", in Proc. of Optical Fiber Commun. Conf., Mar. 2003, pp. 355-357.

[5] B. Mukherjee, C. Ou, H. Zhu, K. Zhu, N. Singhal, and S. Yao, "Traffic grooming in mesh optical networks", in Proc. of Optical Fiber Commun. Conf., Mar. 2004.

[6] X. Cao, V. Anand, and C. Qiao, "Multi-layer versus single-layer optical cross-connect architectures for Waveband-switching," in Proc. of IEEE INFOCOM, June 2004, pp.1830-1840.

[7] M. Li, W. Yao, and B. Ramamurthy, "Same-destination-grouping Vs. end-to-end grouping for Waveband-switching in WDM mesh networks", in Proc. of IEEE ICC, May 2005.

[8] R. Douville, D. Papadimitriou, E. Dotaro, R. Izmailov, A. Kolarov, and J. Drake, "Extensions to generalized MPLS in support of waveband switching," Internet draft, draft-douville-ccamp-gmpls-wavebandextensions-04.txt, June 2003.

[9] P.-H. Ho, J. Tapolcai, and H. T. Mouftah, "Diverse routing for shared-protectoin in survivable optical networks," in Proc. of IEEE Globecom, Dec. 2003, vol. 5, pp. 2519-2523.

[10] H. Zang and B. Mukherjee, "A Review of Routing and Wavelength Assignment Approaches for Wavelength-Routed Optical WDM Networks," SPIE/Kluwer Optical Networks Magazine, Vol. 1, No. 1, pp. 47-60, Jan. 2000.

[11] A. Mokhtar and M. Azizoglu, "Adaptive wavelength routing in all-optical networks," IEEE/ACM Transactions on Networking vol. 6, no. 2, pp. 197-206, Apr. 1998. 